Article

\title{
Nanoporous Carbon Nanofibers Decorated with Platinum Nanoparticles for Non-Enzymatic Electrochemical Sensing of $\mathrm{H}_{2} \mathrm{O}_{2}$
}

\section{Yang Li ${ }^{1, \dagger}$, Mingfa Zhang ${ }^{1, \dagger}$, Xiaopeng Zhang ${ }^{1}$, Guocheng Xie ${ }^{1}$, Zhiqiang Su ${ }^{1, *}$ and Gang Wei ${ }^{2, *}$}

1 State Key Laboratory of Chemical Resource Engineering, Beijing University of Chemical Technology, Beijing 100029, China; E-Mails: liyang1113@hotmail.com (Y.L.); zmingfa@hotmail.com (M.Z.); xiaopeng-zhang@hotmail.com (G.X.); guochengxie3@gmail.com (X.Z.)

2 Hybrid Materials Interface Group, Faculty of Production Engineering, University of Bremen, Bremen D-28359, Germany

$\dagger$ These authors contributed equally to this work.

* Authors to whom correspondence should be addressed; E-Mails: suzq@mail.buct.edu.cn (Z.S.); wei@uni-bremen.de (G.W.); Tel.: +49-421-218-64581 (G.W.).

Academic Editor: Ming Su

Received: 29 August 2015 / Accepted: 30 October 2015 / Published: 6 November 2015

\begin{abstract}
We describe the preparation of nanoporous carbon nanofibers (CNFs) decorated with platinum nanoparticles (PtNPs) in this work by electrospining polyacrylonitrile (PAN) nanofibers and subsequent carbonization and binding of PtNPs. The fabricated nanoporous CNF-PtNP hybrids were further utilized to modify glass carbon electrodes and used for the non-enzymatic amperometric biosensor for the highly sensitive detection of hydrogen peroxide $\left(\mathrm{H}_{2} \mathrm{O}_{2}\right)$. The morphologies of the fabricated nanoporous CNF-PtNP hybrids were observed by scanning electron microscopy, transmission electron microscopy, and their structure was further investigated with Brunauer-Emmett-Teller (BET) surface area analysis, X-ray photoelectron spectroscopy, X-ray diffraction, and Raman spectrum. The cyclic voltammetry experiments indicate that CNF-PtNP modified electrodes have high electrocatalytic activity toward $\mathrm{H}_{2} \mathrm{O}_{2}$ and the chronoamperometry measurements illustrate that the fabricated biosensor has a high sensitivity for detecting $\mathrm{H}_{2} \mathrm{O}_{2}$. We anticipate that the strategies utilized in this work will not only guide the further design and fabrication of functional nanofiber-based biomaterials and nanodevices, but also extend the potential applications in energy storage, cytology, and tissue engineering.
\end{abstract}


Keywords: electrospining; carbon nanofibers; nanoparticles; biosensor; $\mathrm{H}_{2} \mathrm{O}_{2}$

\section{Introduction}

Recently, the electrochemical biosensors have attracted more and more attention for their excellent performance in both basic and applied studies [1-3]. Among all its applications, the analytical determination of hydrogen peroxide $\left(\mathrm{H}_{2} \mathrm{O}_{2}\right)$ is an emerging field [4-6]. Some techniques, such as spectrophotometry [7], chemiluminescence [8], and electrochemistry [9,10] have been employed previously to detect $\mathrm{H}_{2} \mathrm{O}_{2}$. However, electrochemical detection as a novel nonenzymatic detection method of $\mathrm{H}_{2} \mathrm{O}_{2}$, has always been the research focus due to its simplicity and low cost [11].

In order to ensure the detection performance of nonenzymatic electrochemical sensors, a suitable catalyst is necessary $[12,13]$. Generally speaking, metal nanoparticles (MNPs) are the major candidates for the fabrication of electrochemical sensors. Many researches have confirmed that $\mathrm{Au}, \mathrm{Ag}, \mathrm{Pt}, \mathrm{Cu}$, and $\mathrm{Ni}$ nanoparticles possess very good electrochemical activity toward $\mathrm{H}_{2} \mathrm{O}_{2}$ [14-17]. Based on this point, many non-enzymatic electrochemical sensors have been fabricated and used for sensing $\mathrm{H}_{2} \mathrm{O}_{2}$ [18-23]. For example, in our previous work, we prepared the polyurethane nanofibers (PU-NFs) filled with multi-walled carbon nanotubes (MWCNTs) and silver nanoparticles (PU-MWCNT-AgNPs) by electrospinning. Subsequently, a novel non-enzymatic amperometric biosensor was fabricated by these NFs to detect $\mathrm{H}_{2} \mathrm{O}_{2}$ [18]. In another study, Fratoddi et al. prepared a $\mathrm{H}_{2} \mathrm{O}_{2}$ sensor that can work at room temperature by depositing PtNPs onto titania nanofibers ( $\mathrm{TiO}_{2} \mathrm{NFs}$ ) obtained by electrospinning [22]. They found that $\mathrm{Pt}_{-} \mathrm{TiO}_{2}$ hybrid showed combined properties of photoconduction of titania and the photocatalytic activity of the hybrid. Besides, during the sensing tests toward hydrogen, an enhancement of photoconductivity (150\%), an increase in response (400\%), and an overall improvement of dynamic behavior were observed. Among all the MNPs, platinum nanoparticles (PtNPs) play the most important and irreplaceable roles not only for their excellent electrochemical performances but also for their high stability and activity or oxygen reduction reaction (ORR) [24-26]. Previously, we prepared a novel $\beta$-phase polyvinylidene difluoride nanofibrous membrane decorated with MWCNTs and PtNPs [25]. Then, the potential applications as electrode material for the fabrication of $\mathrm{H}_{2} \mathrm{O}_{2}$ and glucose biosensors, as well as for oxidation-reduction reaction (ORR) catalysis were further investigated. It was found that the fabricated biosensors are highly stable and sensitive, and can be used for the selective detection of both $\mathrm{H}_{2} \mathrm{O}_{2}$ and glucose. Additionally, the excellent electrocatalysis as ORR catalyst was also displayed.

Electrospinning is a simple but effective technique to synthesize organized functional polymer nanofibers (NFs) with exceptionally long length, uniform diameter, and large surface area [26-31]. It has been widely used for the preparation of enzyme-based electrochemical sensors previously [32-35]. However, only a few studies on the non-enzymatic electrochemical biosensors based on electrospun NFs on electrodes have been reported [36,37]. Carbon nanofibers (CNFs), the newest product of electrospinning, have attracted increasing attention due to their superior chemical, electrical, and mechanical properties [38-40]. Among various CNFs, nanoporous CNFs exhibit promising applications in energy conversion and storage, gas adsorption, and biomedical engineering, ascribed to their ultrahigh specific surface area and porosity $[41,42]$. 
In this work, we developed a facile strategy to fabricate a novel nanoporous PAN-based CNF decorated with PtNPs (CNF-PtNP) by electrospinning technique, which can serve as the functional material for the fabrication of non-enzymatic $\mathrm{H}_{2} \mathrm{O}_{2}$ biosensor. Firstly, PAN-CaCO 3 NFs were electrospun onto the tinfoil, as show in Figure 1a. During the electrospinning process, the porogen ( $\mathrm{CaCO}_{3}$, nanoscale) can be well dispersed in the created PAN NFs, which can contribute to the formation of NFs with nanoporous structure. After that, PAN-based CNFs were created by the preoxidation and carbonization process, and then nanoporous CNFs were prepared by leaching with $\mathrm{HCl}(2 \mathrm{M})$. Finally, PtNPs were loaded onto the surface of CNF by the reduction of chloroplatinic acid hydrate (Figure 1b). The fabricated three-dimensional (3D) CNF-PtNP membrane with high surface area ratio to volume is beneficial to the adsorption of electrolytes and the diffusion of reactants. As a result, the special porous structure of the CNFs can result in highly stable, sensitive, and selective detection of $\mathrm{H}_{2} \mathrm{O}_{2}$.

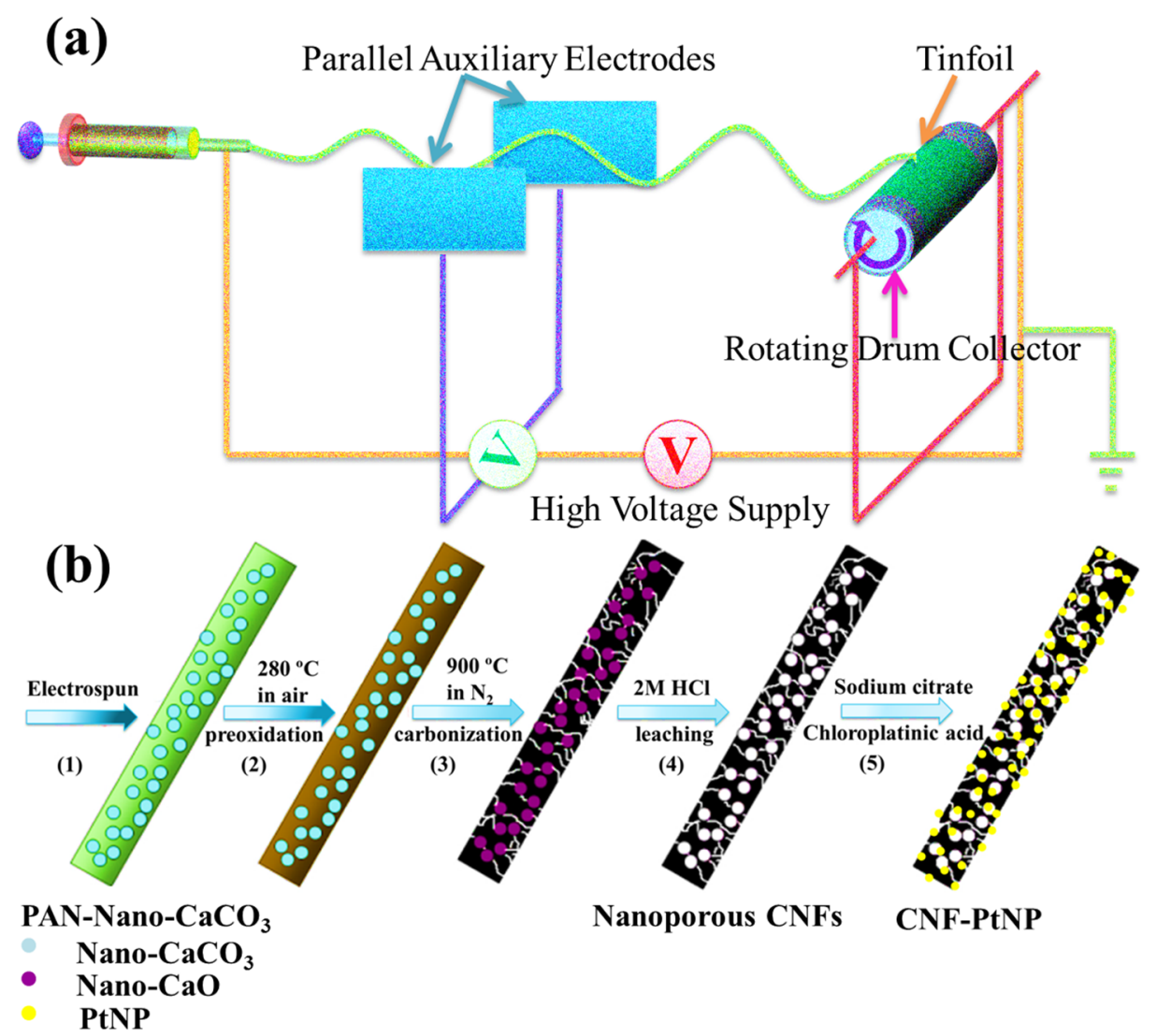

Figure 1. Schematic presentation on: (a) The electrospinning apparatus for preparing the polyacrylonitrile (PAN)-CaCO 3 nanofibers (NFs); and (b) Preparation of nanoporous carbon nanofibers (CNFs) decorated with platinum nanoparticles (PtNPs) (CNF-PtNP) hybrid.

\section{Results and Discussion}

\subsection{Morphologies of Electrospun Nanoporous CNF-PtNP Hybrids}

The morphologies of the electrospun $\mathrm{PAN}-\mathrm{CaCO}_{3}$ NFs were observed firstly by scanning electron microscopy (SEM). It can be clearly seen that the created $\mathrm{PAN}_{-\mathrm{CaCO}} \mathrm{NF}$ are relatively uniform and uniaxial-oriented with smooth surface, as shown in Figure 2a,b. The width of the created NFs is about 150-300 nm. The dispersion of $\mathrm{CaCO}_{3}$ NPs in the electrospun PAN NFs was shown in Figure 2c,d, 
which is the longitudinal section of the NFs. In this direction, the surface of NFs is rough, on which there are many obvious wrinkles. In addition, some particulate matters of $\mathrm{CaCO}_{3} \mathrm{NPs}$ can also be observed with a relative uniform distribution (Figure $2 \mathrm{~d}$ ).

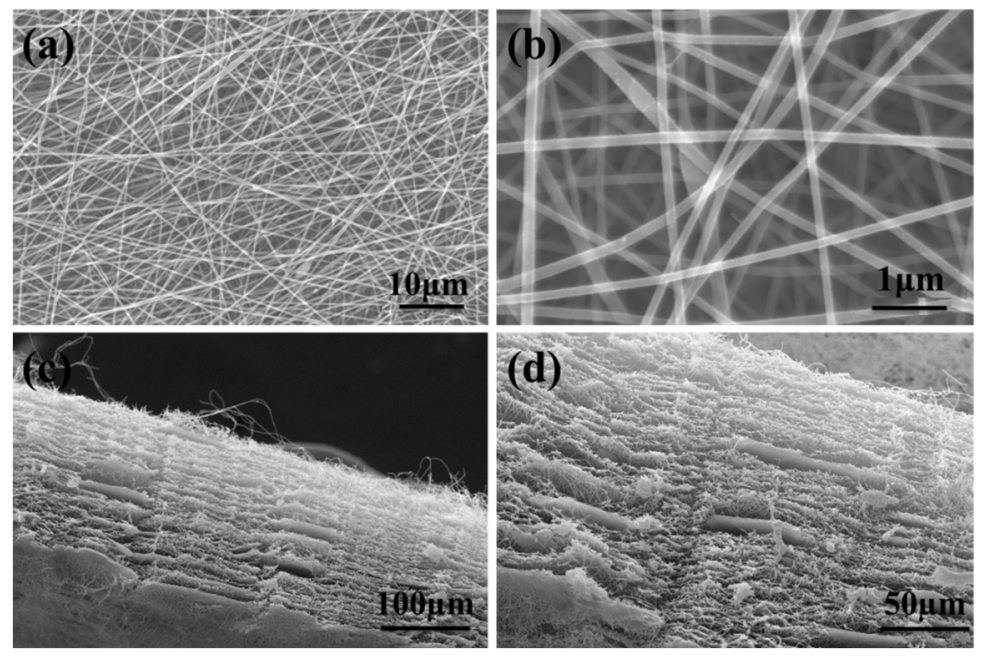

Figure 2. Scanning electron microscopy (SEM) images of electrospun $\mathrm{PAN}-\mathrm{CaCO}_{3} \mathrm{NFs}$ : (a,b) Different magnification, and (c,d) Longitudinal section.

The morphologies of the electrospun $\mathrm{PAN}-\mathrm{CaCO}_{3} \mathrm{NFs}$ after peroxidation and carbonization are shown in Figure 3a. The integrity of the NFs is almost unchanged, which indicates that the PAN NFs didn't undergo the oxidative attack severely. After carbonization, $\mathrm{CaCO}_{3}$ was decomposed into $\mathrm{CaO}$. While the surface of $\mathrm{PAN}-\mathrm{CaCO}_{3} \mathrm{NFs}$ is much rougher with many $\mathrm{CaO}$ nodules distributed throughout the NFs, the distribution of the NFs diameter becomes larger, ranging from about 200 to $400 \mathrm{~nm}$. Due to the high elongation of polymer jet during the electrospinning process, the $\mathrm{CaCO}_{3}$ shows a little aggregation and heterogeneous distribution along the fiber axis, as shown in the red circle in Figure $3 \mathrm{a}$. In fact, this kind of $\mathrm{CaCO}_{3}$ can be eroded by $\mathrm{HCl}$. Figure $3 \mathrm{~b}$ shows the typical SEM images of the fabricated nanoporous CNFs, which have been leached by $\mathrm{HCl}$. It can be clearly seen that all the fabricated NFs are highly porous, and the leaching by $\mathrm{HCl}$ cannot affect the diameter and length of the electrospun NFs [43]. However, due to the difference of the porogen aggregation, the size of nano-pores embedded in the NFs is not very uniform. The cross-section of the porous CNFs was shown in Figure 3c. As we can see in this figure, the porous structure is very clear. The aggregation of nano- $\mathrm{CaCO}_{3}$ in the fiber interior caused by electrospinning might be the source of pores. It should be noted that although the porous structure observed by SEM is not very uniform, the dispersion of nano- $\mathrm{CaCO}_{3}$ in the PAN matrix was relatively homogeneous. Figure $3 d$ shows the typical TEM images of the nanoporous CNFs, and it was found that the morphology of the porous structure is very clear. Nitrogen adsorption-desorption isotherm analysis was further performed to evaluate the surface area of the created CNF-PtNP hybrids (Figure 4). The Brunauer-Emmett-Teller (BET) measurement suggests a mecro/mesoporous structure of the created nano-CNFs, as evidenced by the nitrogen adsorption/desorption isotherms of IV type. Based on the BET analysis, the specific surface area of the porous nano-CNFs is calculated to be $131.7 \mathrm{~m}^{2} / \mathrm{g}$, which is beneficial to the electrochemical performance [44]. 

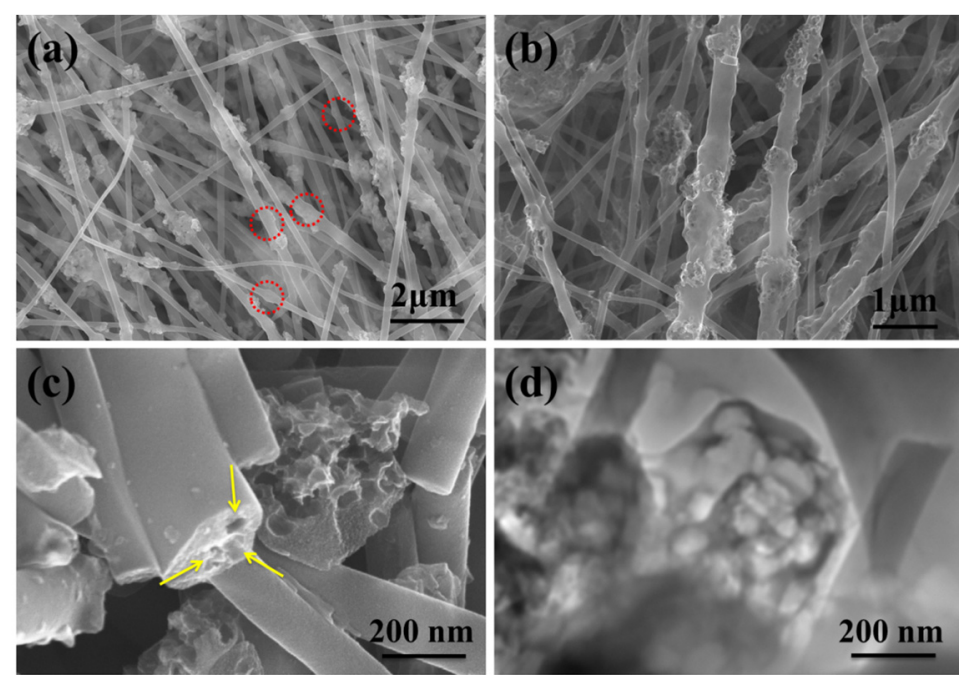

Figure 3. Morphology characterization: (a,b) SEM images of electrospun $\mathrm{PAN}-\mathrm{CaCO}_{3}$ hybrid NFs by treating with carbonization and $\mathrm{HCl}$; (c) SEM image for the cross-section of porous CNFs; (d) TEM image of the porous CNFs.

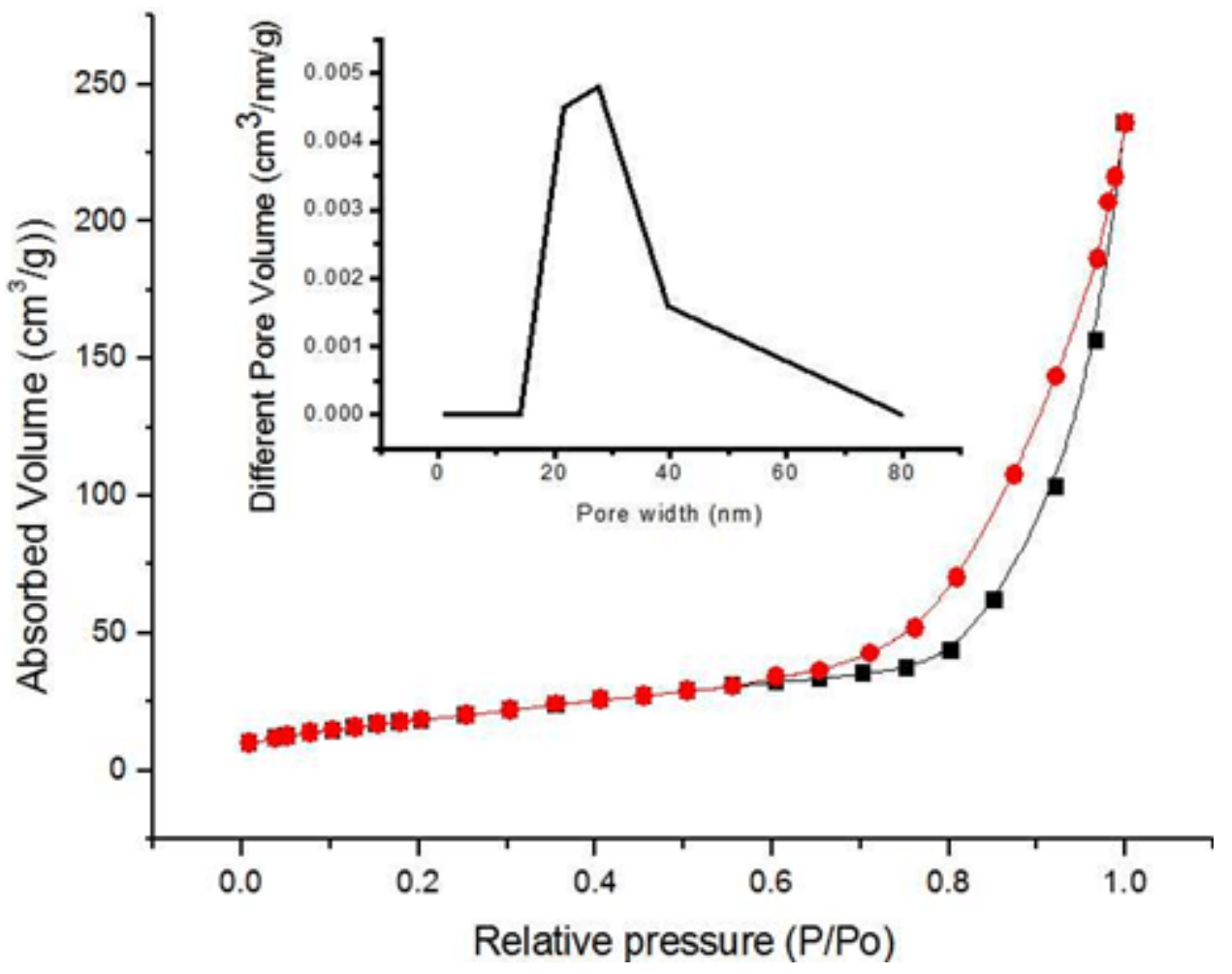

Figure 4. $\mathrm{N}_{2}$ adsorption/desorption isotherms of the CNF-PtNP hybrids as well as the pore size distributions.

\subsection{Structural Characterization of Nanoporous CNF-PtNP Hybrids}

Figure 5a shows the SEM image of the created nanoporous CNF-PtNP hybrids. It can be found that the PtNPs were bound onto the surface of CNFs uniformly due to the gentle redox reactions between citric acid and chloroplatinic acid hydrate. The previously generated holes increase the surface area of CNFs dramatically, and therefore cause the adhesion of a large amount of PtNPs onto the CNFs. The 
typical energy-dispersive X-ray (EDX) spectrum of the fabricated nanoporous CNF-PtNP hybrid is shown in Figure 5b. Two characteristic peaks at about 2.2 and $9.4 \mathrm{KeV}$ on the EDX spectra can be observed clearly, which identifies the successful Pt adsorption on CNFs. By the analysis and calculation of EDX, we can obtain that the adsorption amount of Pt is $8.31 \%$, which is a fairly good adsorption.
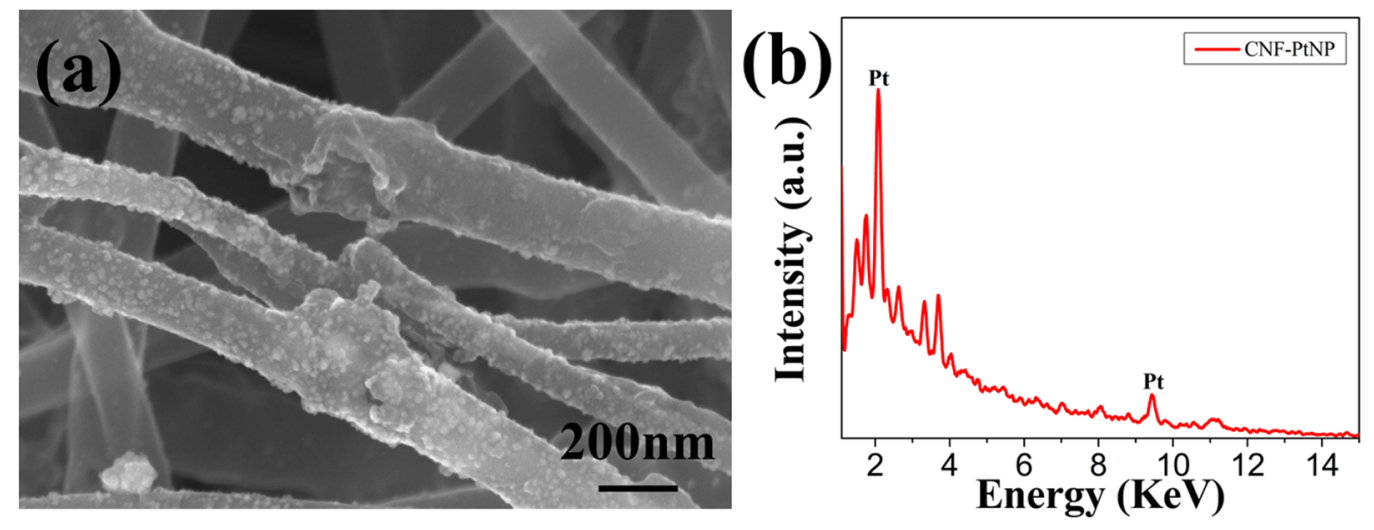

Figure 5. (a) Typical SEM image of the fabricated nanoporous CNF-PtNP hybrids; (b) Energy-dispersive X-ray (EDX) spectra of the fabricated nanoporous CNF-PtNP hybrids.

To identify the existence of PtNPs in the created CNFs, X-ray photoelectron spectroscopy (XPS) was performed firstly. Figure 6a shows the obtained XPS spectrum of the decorated CNFs from 0 to $700 \mathrm{eV}$. Four different peaks are observed, corresponding to the elemental Pt, C, N and O, respectively. Obviously, the existence of $\mathrm{N}(9.14 \%)$ is the remnant of PAN during the carbonization [45]. To make the peaks of Pt more distinguishable, we carried another XPS analysis with an abscissa from 64 to $80 \mathrm{eV}$. As a result, two significant characteristic peaks at 71 and $74.3 \mathrm{eV}$ can be seen, which are assigned to the $4 \mathrm{f}_{7 / 2}$ and the $4 \mathrm{f}_{5 / 2}$ planes of the embedded PtNPs [46], respectively. Based on the above XPS results, we suggest that the CNFs are indeed decorated by PtNPs.

Power X-ray diffraction (XRD) was further used to prove the formation of CNF-PtNP nanostructure, and the typical pattern is shown in Figure 6c. Obviously, the peak at $20.2^{\circ}$ (C element) of the electrospun NFs is of strongest intensity compared to other peaks. The peaks reveal characteristic reflections of $\mathrm{Pt}$ at $39.7^{\circ}, 46.2^{\circ}$, and $67.5^{\circ}$, which could be assigned to the (111), (200), and (220) planes of the embedded PtNPs, respectively [47] (JCPDS no. 87-0640). Therefore, we can further prove that the electrospun NFs are really the CNF-PtNP hybrids. Figure 6d shows the corresponding Raman spectrum of the fabricated CNF-PtNP hybrids. Two characteristic peaks (D and G bands) at about 1363 and $1594 \mathrm{~cm}^{-1}$ [48] can be observed clearly, which identifies the successful carbonization of PAN NFs and the formation of CNFs. 

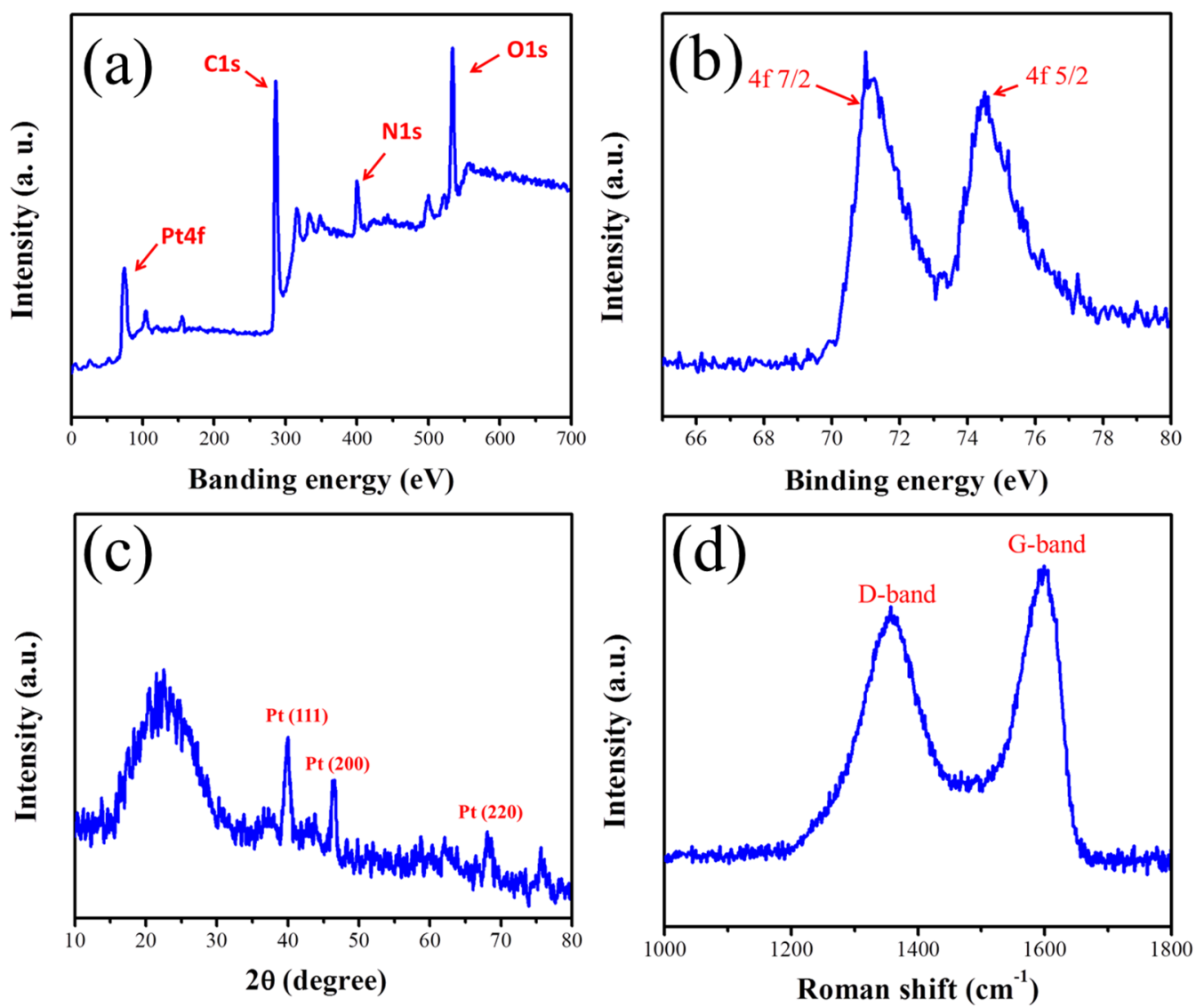

Figure 6. Characterization of electrospun CNF-PtNP hybrids: $(\mathbf{a}, \mathbf{b}) \mathrm{X}$-ray photoelectron spectroscopy (XPS) spectra; (c) Power X-ray diffraction (XRD) pattern; and (d) Raman spectrum.

\subsection{Non-Enzymatic Electrochemical Detection of $\mathrm{H}_{2} \mathrm{O}_{2}$}

Considering the good electrocatalytic performance of CNF-PtNP hybrids, as well as their excellent nanoporous structure, the prepared CNF-PtNP hybrids are expected to show good performance for electrochemical sensors. To test this hypothesis, CNF-PtNP hybrids were utilized as the electrode materials to modify the glass carbon electrode (GCE), and the fabricated sensor platform was further utilized for the detection of $\mathrm{H}_{2} \mathrm{O}_{2}$ in this work. To discern the importance of PtNPs on the sensor performances, control experiments with bare GCE, CNF/GCE, and CNF-PtNP/GCE were also carried out.

Figure 7a displays the typical cyclic voltammograms (CVs) of bare GCE, CNF/GCE, and CNF-PtNP/GCE in the presence of $5 \mathrm{mM} \mathrm{H}_{2} \mathrm{O}_{2}$. It is clear that both bare GCE and CNF/GCE show no redox processes, while the CNF-PtNP/GCE demonstrates a reduction peak at about $0.4 \mathrm{~V}$ with an obvious positive shift for both the onset potential and current peak. Therefore, the present CV results indicate that the CNF-PtNP/GCE has better electrocatalytic activity than other NFs modified GCE toward the reduction of $\mathrm{H}_{2} \mathrm{O}_{2}$, which confirms the importance of PtNPs. To further check the electrocatalytic performance of CNF-PtNP/GCE toward $\mathrm{H}_{2} \mathrm{O}_{2}$, the chronoamperometry of the CNF-PtNP/GCE were also performed at an applied potential of $-0.34 \mathrm{~V} v s$. SCE. As shown in Figure $7 \mathrm{~b}$, a stable response over the long period test and rapid increase in the cathodic current as a result of the reduction of $\mathrm{H}_{2} \mathrm{O}_{2}$ upon adding $\mathrm{H}_{2} \mathrm{O}_{2}$ solutions with different concentrations are observed. The corresponding calibration curve indicates that the sensor shows two linear regions for the response to $\mathrm{H}_{2} \mathrm{O}_{2}$ in the ranges 
of $10 \mu \mathrm{M}$ to $9.38 \mathrm{mM}$ and 9.38 to $74.38 \mathrm{mM}$, as shown in Figure 7c. Based on the first linear range, the line arregression equation of $I(\mu \mathrm{A})=-2.2506-1.0444 C(\mathrm{mM})\left(R^{2}=0.9432\right)$ is determined. In a higher concentration of $\mathrm{H}_{2} \mathrm{O}_{2}$, the second linear section raised up to $90 \mathrm{mM}$ (Figure $6 \mathrm{c}$ ) with a linear equation of $I(\mu \mathrm{A})=-10.3417-0.1862 C(\mathrm{mM})\left(R^{2}=0.9395\right)$. The limit of detection $(L O D)$ of CNF-PtNP/GCE biosensor was determined by using the following Equations [49]:

$$
L O D=3 \sigma / S
$$

where $\sigma$ is the standard deviation of the response, and $S$ is the slope of the calibration curve.

Compared to previous reports toward the $\mathrm{H}_{2} \mathrm{O}_{2}$ sensor [50], our $\mathrm{H}_{2} \mathrm{O}_{2}$ biosensor based on CNF-PtNP has a similar low $L O D$ of about $1.9 \mu \mathrm{M}$. Besides, comparing the results shown in Figure $7 \mathrm{a}$, it can be clearly found that the onset potential for $\mathrm{H}_{2} \mathrm{O}_{2}$ reduction in the CNF-PtNP/GCE appears more positive than its counterpart electrode, which reveals the superiority of the electrospun NFs in electrochemical sensing of $\mathrm{H}_{2} \mathrm{O}_{2}$.
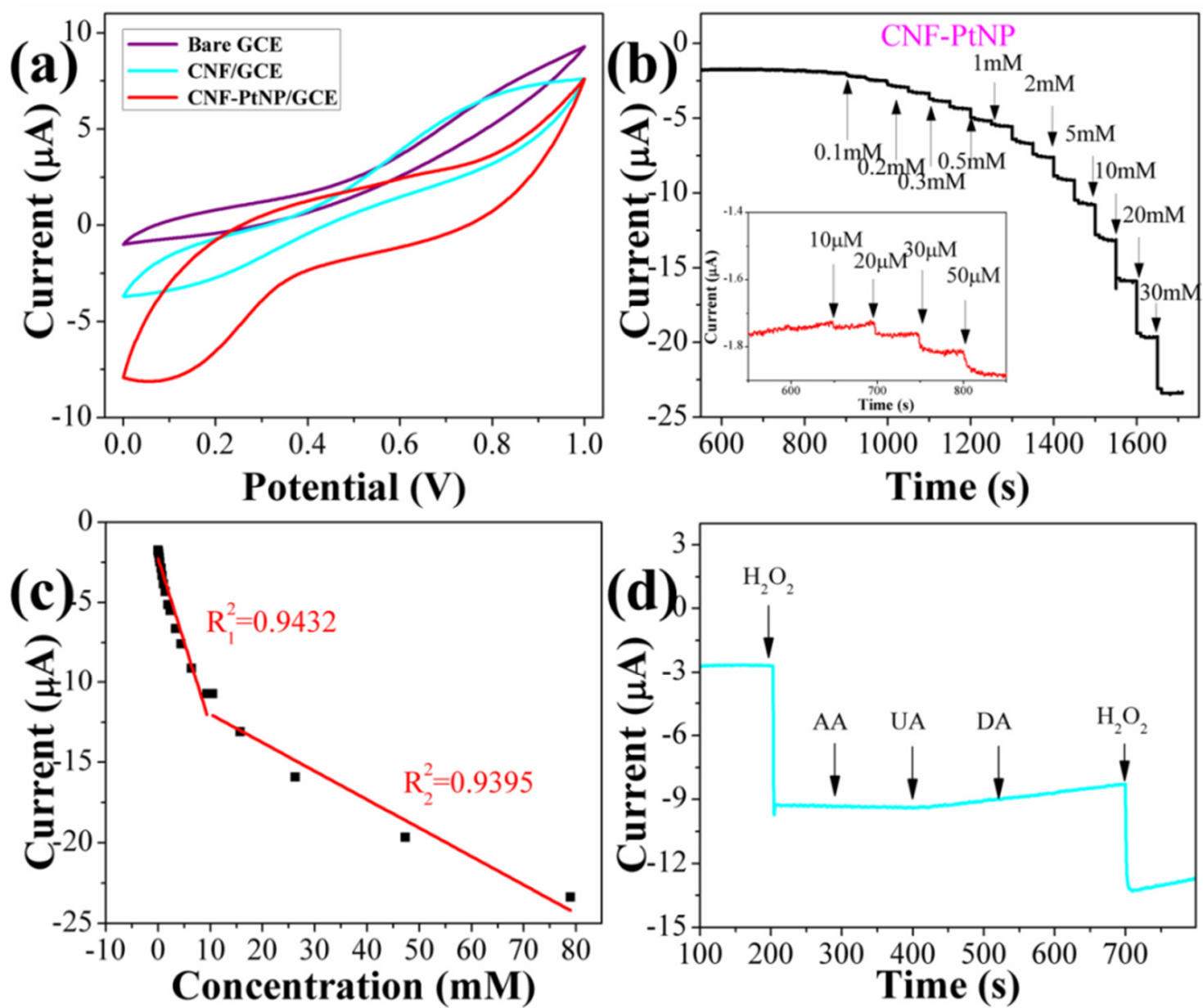

Figure 7. Electrochemical detection of $\mathrm{H}_{2} \mathrm{O}_{2}$ : (a) Cyclic voltammograms (CVs) of glass carbon electrode (GCE) and GCEs modified with CNFs and CNF-PtNP hybrids; (b) I-T response of CNF-PtNP modified GCE; (c) Calibrated line; and (d) Selectivity of biosensor.

Finally, the selectivity and anti-interference capability of the CNF-PtNP/GCE sensor platform were evaluated by amperometry in the presence of ascorbic acid (AA), uric acid (UA), dopamine (DA), which are the common interfering substances for the detection of $\mathrm{H}_{2} \mathrm{O}_{2}$. As shown in Figure $7 \mathrm{~d}$, the 
amperometric responses of the CNF-PtNP/GCE for the successive addition of $1 \mathrm{mM}$ of $\mathrm{H}_{2} \mathrm{O}_{2}, 0.1 \mathrm{mM}$ of AA, $0.10 \mathrm{mM}$ of UA, $0.10 \mathrm{mM}$ of DA, and $1 \mathrm{mM}$ of $\mathrm{H}_{2} \mathrm{O}_{2}$ were presented. It can be found that the interferences with these species on the detection of $\mathrm{H}_{2} \mathrm{O}_{2}$ are negligible, which means that CNF-PtNP/GCE possesses a high selectivity toward $\mathrm{H}_{2} \mathrm{O}_{2}$.

Based on the above results and discussions, we can deduce that the superiority of this non-enzymatic sensor is ascribed to the advantages of the electrospinning technique. During the electrospinning process, the nanoscale $\mathrm{CaCO}_{3}$ can be successfully dispersed and aligned along the NFs, making the fabricated CNFs highly porous, as can be seen from the SEM photographs (Figure 2). In addition, this nanoporous structure can greatly increase the effective adhesion area of PtNPs and further enhance the electrocatalytic activity of electrodes.

\section{Experimental Section}

\subsection{Reagents and Materials}

Calcium carbonate (nano- $\mathrm{CaCO}_{3}$ ) was purchased from Shanxi Xintai Hengxin NanoMaterials Technology Co., Ltd (Shanxi, China). Polyacrylonitrile (PAN) ( $M w=150,000$, J\&K Scientific Ltd., Beijing, China). $N, N$-Dimethylformamide (DMF, $>99.8 \%$ purity) was obtained from Aladdin (Shanghai, China). Sodium citrate tribasic dehydrate ( $\geq 99.0 \%$ purity), chloroplatinic acid hydrate ( $\geq 99.9 \%$ purity), and Nafion solution ( $\sim 5 \%$ in a mixture of lower aliphatic alcohols and water) were purchased from Sigma-Aldrich (St. Louis, MO, USA). Disodium hydrogen phosphate $\left(\mathrm{Na}_{2} \mathrm{HPO}_{4}\right)$, sodium dihydrogen phosphate $\left(\mathrm{NaH}_{2} \mathrm{PO}_{4}\right)$, ethanol, AA, UA, and DA were purchased from Beijing Chemicals Co., Ltd. (Beijing, China). $\mathrm{H}_{2} \mathrm{O}_{2}$ (analytical grade, $30 \%$ aqueous solution) was supplied by Tianjin Dong fang Chemical Plant (Tianjin, China). All chemicals used in this work were analytical reagents and obtained from commercial sources and directly used without additional purification. The water used was purified through a Millipore system $(\sim 18.2 \mathrm{M} \Omega \cdot \mathrm{cm})$.

\subsection{Preparation of Nanoporous CNFS}

To uniformly generate holes in the PAN matrix, nano- $\mathrm{CaCO}_{3}$ was firstly dispersed in DMF using an ultrasonic cleaner operating at $40 \mathrm{kHz}$ for $30 \mathrm{~min}$, respectively. The resulting dispersions were homogeneous and stable. Meanwhile, PAN was dissolved in the mixture of nano- $\mathrm{CaCO}_{3}$ and DMF to form a uniform transparent solution by using an ultrasonic cleaner operating at $40 \mathrm{kHz}$ for $1 \mathrm{~h}$. The concentrations of PAN were $3 \mathrm{wt} \%$ in the solutions. The dispersions were then loaded into plastic $10 \mathrm{~mL}$ syringes with an 18-gauge blunt tip needle and were dispensed at a rate of $0.1-0.3 \mathrm{~mL} / \mathrm{h}$ during electrospinning. All the samples were electrospun with an applied voltage of $12 \mathrm{kV}$ at a distance of $12 \mathrm{~cm}$ from the needle tip to the collector surface of tinfoil. The as-spun PAN NFs were treated by peroxidation at $280^{\circ} \mathrm{C}$ in air (at a rate of $2{ }^{\circ} \mathrm{C} \cdot \mathrm{min}^{-1}$ and keeping this temperature for $2 \mathrm{~h}$ ). Then a high temperature furnace was employed to stabilize and carbonize the PAN NFs at $900{ }^{\circ} \mathrm{C}$ in $\mathrm{N}_{2}$ atmosphere (at a rate of $5^{\circ} \mathrm{C} \cdot \mathrm{min}^{-1}$ to carbonize the NFs, keeping the highest temperature for $2 \mathrm{~h}$ ). At this time, the nano- $\mathrm{CaCO}_{3}$ had been decomposed into the calcium oxide (nano-CaO). Last, $\mathrm{HCl}$ was used to leach the NFs. The nano-CaO was dissolved and the porous structure was formed. 


\subsection{Preparation of Nanoporous CNF-PtNP Hybrids}

A typical process was employed to load the PtNPs onto the surface of PAN-based nanoporous CNF. In brief, $50 \mathrm{mg} \mathrm{CNF}$ and $30 \mathrm{~mL}$ chloroplatinic acid hydrate $(0.03 \mathrm{mM})$ were added in a $50 \mathrm{~mL}$ flask under a gentle stirring in $\mathrm{N}_{2}$ atmosphere at $80{ }^{\circ} \mathrm{C}$, followed by adding $10 \mathrm{~mL}$ aqueous sodium citrate (0.1 wt \%) react for $1 \mathrm{~h}$ to obtain CNF-PtNP. After that, the products were washed with ethanol and distilled water in an ultrasonic bath. Finally, the fabricated nanoporous CNF-PtNP hybrids were dried at room temperature.

\subsection{Preparation of CNF-PtNP Modified GCE}

The procedure for the fabrication of the CNF-PtNP/GCE is as follows: First, $10 \mathrm{mg}$ CNF was added into $10 \mathrm{~mL}$ phosphate buffer $(\mathrm{pH}=7.6)$ to obtain $\mathrm{CNF}$ suspension under the condition of ultrasonication and stirring. Next, a mixture was created containing $1 \mathrm{~mL}$ Nafion (5 wt \%) and $2 \mathrm{~mL}$ CNF-PtNP suspension, and the mixture was kept stirring for $1 \mathrm{~h}$. Lastly, the CNF-PtNP/GCE was prepared by dropping $10 \mu \mathrm{L}$ of the mixture onto the surface of a freshly polished glass carbon electrode. The GCE was polished with 1 and $0.3 \mu \mathrm{m}$ alumina slurry before the modification and then successively washed with ethanol and distilled water in an ultrasonic bath for $10 \mathrm{~s}$ respectively. Finally, the fabricated CNF-PtNP/GCEs were dried in air for biosensor application. The dried CNF-PtNP/GCE was kept in storage at $4{ }^{\circ} \mathrm{C}$. Meanwhile, brae GCE and CNF/GCE were prepared as the control experimental. In addition, the mass of CNF and nafion in different electrodes should be kept equal.

\subsection{Characterization Techniques}

SEM morphologies of the electrospun NFs were performed on a JSM-6700F scanning electron microscope (JEOL, Tokyo, Japan) at $20 \mathrm{kV}$. TEM images were taken by a Tecnai G220 transmission electron microscope (FEI, Beijing, China) with an accelerating voltage of $200 \mathrm{kV}$, and samples were prepared by directly electrospinning on to the copper grid X-ray diffraction (XRD, Rigaku D/max-2500 VB+/PC, Shanghai, China), X-ray photoelectron spectroscopy (XPS, ThermoVG ESCALAB 250, Tokyo, Japan), and Raman spectroscopy (LabRAM HORIBA JY, Edison, NJ, USA) were used to characterize the structure of samples. The Brunauer-Emmett-Teller (BET, Beijing, China) surface areas of the obtained porous CNFs were analyzed by using nitrogen adsorption in a Micromeritics 3H-2000PS1 nitrogen adsorption apparatus (BET, Beijing, China).

\subsection{Electrochemical Experiments}

All electrochemical experiments were performed on a $\mathrm{CHI}$ 660A electrochemical workstation (CH Instruments, Shanghai, China) at room temperature. A conventional three-electrode system was employed with a bare or modified GCE as the working electrode, a Pt wire as the auxiliary electrode, and a $\mathrm{KCl}$ saturated calomel electrode ( $\mathrm{SCE}$ ) as the reference electrode. The test solutions were phosphate buffer solutions ( $\mathrm{PBS}, 0.1 \mathrm{M}, \mathrm{pH}=7.6$ ), which were prepared with $0.1 \mathrm{M} \mathrm{NaH}_{2} \mathrm{PO}_{4}$ and $0.1 \mathrm{M} \mathrm{Na}_{2} \mathrm{HPO}_{4}$ and deoxygenated with highly pure nitrogen for $20 \mathrm{~min}$ before electrochemical experiments. All potentials in this work refer to the SCE. The curves of CVs in this work were obtained 
after six repetitions of scan numbers under steady-state conditions. Amperometric measurements were carried out under stirred conditions.

\section{Conclusions}

In summary, we demonstrated a facile and efficient electrospinning technique to fabricate novel nanoporous CNFs decorated with PtNPs, and further investigated potential applications, such as $\mathrm{H}_{2} \mathrm{O}_{2}$ biosensors. Electrochemical data indicate that the CNF-PtNP based sensors show good electrocatalytic activity toward $\mathrm{H}_{2} \mathrm{O}_{2}$. The fabricated biosensor shows a wide linear range, low detection limitation, and high selectivity due to the uniform porous structure of CNF, as well as the even coverage of nanoparticles, which are thought to be the main reasons to promote the electrochemical and electrocatalysis properties of the electrospun CNF-PtNP hybrids. We believe that the electrospinning technique can be utilized to prepare nanoporous multifunctional nanomaterials by introducing different functional nanomaterials or building blocks into the polymer NFs as this will be very helpful in fabricating other kinds of sensors with wide applications in actuators, generators, water purification, and energy storage.

\section{Acknowledgments}

We acknowledge the financial support from the National Natural Science Foundation of China (Grant No. 51573013).

\section{Author Contributions}

Z.S. and G.W. supervised the entire project. Y.L. and M.Z. performed the characterizations of materials and electrochemical experiments. G.X. and X.Z. assisted part of the experiments. Y.L. and M.Z. co-wrote the paper.

\section{Conflicts of Interest}

The authors declare no conflict of interest.

\section{References}

1. Zhang, P.P.; Zhao, X.N.; Ji, Y.C.; Ouyang, Z.F.; Wen, X.; Li, J.F.; Wei, G.; Su, Z.Q. Electrospinning graphene quantum dots into nanofibrous polymer membrane for dual-purpose fluorescent and electrochemical biosensors. J. Mater. Chem. B 2015, 3, 2487-2496.

2. Ding, J.W.; Zhu, S.Y.; Zhu, T.; Sun, W.; Li, Q.; Wei, G.; Su, Z.Q. Hydrothermal synthesis of zinc oxide-reduced graphene oxide nanocomposites for an electrochemical hydrazine sensor. RSC Adv. 2015, 5, 22935-22942.

3. Zhang, M.F.; Li, Y.; Su, Z.Q.; Wei, G. Recent advances in the synthesis and applications of graphene-polymer nanocomposites. Polym. Chem. 2015, 6, 6107-6124.

4. Fang, H.; Pan, Y.; Shan, W.; Guo, M.; Nie, Z.; Huang Y.; Yao, S. Enhanced nonenzymatic sensing of hydrogen peroxide released from living cells based on $\mathrm{Fe}_{3} \mathrm{O}_{4} /$ self-reduced graphene nanocomposites. Anal. Methods 2014, 6, 6073-6081. 
5. Lee, K.T.; Liu, D.M.; Liang, Y.Y.; Matsushita, N.; Ikoma, T.; Lu, S.Y. Porous fluorine-doped tin oxide as a promising substrate for electrochemical biosensors-demonstration in hydrogen peroxide sensing. J. Mater. Chem. B 2014, 2, 7779-7784.

6. Ding, J.W.; Sun, W.; Wei, G.; Su, Z.Q. Cuprous oxide microspheres on graphene nanosheet: An enhanced material for non-enzymatic electrochemical detection of $\mathrm{H}_{2} \mathrm{O}_{2}$ and glucose. RSC Adv. 2015, 5, 35338-35345.

7. Eren, S.; Üzer, A.; Can, Z.; Kapudan, T.; Erçă̆, E.; Apak, R. Determination of peroxide-based explosives with copper (II)-neocuproine assay combined with a molecular spectroscopic sensor. Analyst 2010, 135, 2085-2091.

8. Lebiga, E.; Fernandez, R.E.; Beskok, A. Confined chemiluminescence detection of nanomolar levels of $\mathrm{H}_{2} \mathrm{O}_{2}$ in a paper-plastic disposable microfluidic device using a smartphone. Analyst 2015, 140, 5006-5011.

9. Katsounaros, I.; Schneider, W.B.; Meier, J.C.; Benedikt, U.; Biedermann, P.U.; Auer, A.A.; Mayrhofer, K.J.J. Hydrogen peroxide electrochemistry on platinum: Towards understanding the oxygen reduction reaction mechanism. Phys. Chem. Chem. Phys. 2012, 14, 7384-7391.

10. Lv, C.J.; Di, W.H.; Liu, Z.H; Zheng, K.Z.; Qin, W.P. Luminescent CePO4: Tb colloids for $\mathrm{H}_{2} \mathrm{O}_{2}$ and glucose sensing. Analyst 2014, 139, 4547-4555.

11. Wang, G.F.; He, X.P.; Wang, L.L.; Gu, A.X.; Huang, Y.; Fang, B.; Geng, B.Y.; Zhang, X.J. Non-enzymatic electrochemical sensing of glucose. Microchim. Acta 2013, 180, 161-186.

12. Wang, J.H.; Zhao, X.J.; Li, J.F.; Fan, Y.Q.; Kuang, X.; Su, Z.Q.; Wei, G. Electrostatic assembly of peptide nanofiber-biomimetic silver nanowires onto graphene for electrochemical sensors. ACS Macro. Lett. 2014, 3, 529-533.

13. Ding, J.W.; Zhang, K.; Xu, W.; Su, Z.Q. Self-assembly of gold nanoparticles on gold core-induced polypyrrole nanohybrids for electrochemical sensor of dopamine. Nano 2015, doi:10.1142/S1793292015501155.

14. Shironita, S.; Sakai, T.; Umeda, M. Nafion thickness dependence of $\mathrm{H}_{2} \mathrm{O}_{2}$ yield during $\mathrm{O}_{2}$ reduction at Nafion/Pt microelectrode studied by scanning electrochemical microscopy. Electrochim. Acta 2013, 113, 773-778.

15. Li, Y.; Xu, M.; Li, P.; Dong, J.; Ai, S. Nonenzymatic sensing of methyl parathion based on graphene/gadolinium Prussian Blue analogue nanocomposite modified glassy carbon electrode. Anal. Methods 2014, 6, 2157-2162.

16. Zhai, D.; Liu, B.; Shi, Y.; Pan, L.; Wang, Y.; Li, W.; Zhang, R.; Yu, G. Highly sensitive glucose sensor based on pt nanoparticle/polyaniline hydrogel heterostructures. ACS Nano 2013, 7, 3540-3546.

17. Ding, J.W.; Zhang, K.; Wei, G.; Su, Z.Q. Fabrication of polypyrrole nanoplates decorated with silver and gold nanoparticles for sensor applications. RSC Adv. 2015, 5, 69745-69752.

18. Ouyang, Z.F.; Li, J.F.; Wang, J.H.; Li, Q.; Ni, T.Y.; Zhang, X.Y.; Wang, H.X.; Li, Q.; Su, Z.Q.; Wei, G. Fabrication, characterization and sensor application of electrospun polyurethane nanofibers filled with carbon nanotubes and silver nanoparticles. J. Mater. Chem. B 2013, 1, 2415-2424.

19. Wei, G.; Zhang, Y.; Steckbeck, S.; Su, Z.Q.; Li, Z. Biomimetic graphene-FePt nanohybrids with high solubility, ferromagnetism, fluorescence, and enhanced electrocatalytic activity. J. Mater. Chem. 2012, 22, 17190-17195. 
20. Zhang, P.P.; Huang, Y.; Lu, X.; Zhang, S.Y.; Li, J.F.; Su, Z.Q.; Wei, G. One-step synthesis of large-scale graphene film doped with gold nanoparticles at liquid-air interface for electrochemistry and raman detection applications. Langmuir 2014, 30, 8980-8989.

21. Wang, L.; Lu, X.P.; Wen, C.J.; Xie, Y.Z.; Miao, L.F.; Chen, S.H.; Li, H.B.; Li P.; Song, Y.H. One-step synthesis of $\mathrm{Pt}-\mathrm{NiO}$ nanoplate array/reduced graphene oxide nanocomposites for nonenzymatic glucose sensing. J. Mater. Chem. A 2015, 3, 608-616.

22. Fratoddi, I.; Macagnano, A.; Battocchio, C.; Zampetti, E.; Venditti, I.; Russo, M.V.; Bearzotti, A. Platinum nanoparticles on electrospun titania nanofibers as hydrogen sensing materials working at room temperature. Nanoscale 2014, 6, 9177-9184.

23. Li, Y.; Zhao, X.J.; Zhang, P.P.; Ning, J.; Li, J.F.; Su, Z.Q.; Wei, G. A facile fabrication of large-scale reduced graphene oxide-silver nanoparticle hybrid film as a highly active surface-enhanced Raman scattering substrate. J. Mater. Chem. C 2015, 3, 4126-4133.

24. Zhao, R.P.; Liu, Y.; Liu, C.; Xu, G.R.; Chen, Y.; Tang, Y.W.; Lu, T.H. Pd@Pt core-shell tetrapods as highly active and stable electrocatalysts for the oxygen reduction reaction. J. Mater. Chem. A 2014, 2, 20855-20860.

25. Zhang, P.P.; Zhao, X.N.; Zhang, X.; Lai, Y.; Wang, X.Y.; Li, J.F.; Su, Z.Q.; Wei, G. Electrospun doping of carbon nanotubes and platinum nanoparticles into $\beta$-phase polyvinylidene difluoride nanofibrous membrane for biosensor and catalysis applications. ACS Appl. Mater. Interfaces 2014, 6, 7563-7571.

26. Su, Z.Q.; Ding, J.W.; Wei, G. Electrospinning: A facile technique for fabricating polymeric nanofibers doped with carbon nanotubes and metallic nanoparticles for sensor application. RSC Adv. 2014, 4, 52598-52610.

27. Cao, L.; Su, D.F.; Su, Z.Q.; Chen, X.N. Fabrication of multi-walled carbon nanotube/polypropylene conductive fibrous membranes by melt electrospinning. Ind. Eng. Chem. Res. 2014, 53, 2308-2317.

28. Su, Z.Q.; Li, J.F.; Ouyang, Z.F.; Matthias, A.; Wei, G.; Jandt, K.D. Biomimetic 3D hydroxyapatite architectures with interconnected nanopores based on electrospun biaxially orientated PCL nanofibers. RSC Adv. 2014, 4, 14833-14839.

29. Cao, L.; Dong, M.; Zhang, A.Y.; Liu, Y.; Yang, W.M.; Su, Z.Q.; Chen, X.N. Morphologies and crystal structures of styrene-acrylonitrile/isotactic polypropylene ultrafine fibers fabricated by melt electrospinning. Polym. Eng. Sci. 2013, 53, 2674-2682.

30. Sahay, R.; Kumar, P.S.; Sridhar, R.; Sundaramurthy, J.; Venugopal, J.; Mhaisalkar, S.G.; Ramakrishna, S. Electrospun composite nanofibers and their multifaceted applications. J. Mater. Chem. 2012, 22, 12953-12971.

31. Cao, L.; Su, D.F.; Su, Z.Q.; Chen, X.N. Morphology, crystallization behavior and tensile property of beta-nucleated isotactic polypropylene fibrous membranes prepared by melt electrospinning. Chin. J. Polym. Sci. 2014, 32, 1167-1175.

32. Zhang, P.P.; Wang, H.X.; Zhang, X.Y.; Xu, W.; Li, Q.; Wei, G.; Su, Z.Q. Graphene film doped with silver nanoparticles: Self-assembly formation, structural characterizations, antibacterial ability, and biocompatibility. Biomater. Sci. 2015, 3, 852-860. 
33. Mercante, L.A.; Pavinatto, A.; Iwaki, L.E.O.; Scagion, V.P.; Zucolotto, V.; Oliveira, O.N., Jr.; Mattoso, L.H.C.; Correa, D.S. Electrospun polyamide 6/poly(allylamine hydrochloride) nanofibers functionalized with carbon nanotubes for electrochemical detection of dopamine. ACS Appl. Mater. Interfaces 2015, 7, 4784-4790.

34. Zhang, P.P.; Zhang, X.Y.; Zhang, S.Y.; Liu, X.; Li, Q.; Su, Z.Q.; Wei, G. One-pot green synthesis, characterizations, and biosensor application of self-assembled reduced grapheme oxide/gold nanoparticle hybrid membranes. J. Mater. Chem. B 2013, 1, 6525-6531.

35. Wu, J.; Wang, N.; Zhao, Y.; Jiang, L. Electrospinning of multilevel structured functional micro-/nanofibers and their applications. J. Mater. Chem. A 2013, 1, 7290-7305.

36. Pinto, S.C.; Rodrigues, A.R.; Saraiva, J.A.; Lopes-da-Silva, J.A. Catalytic activity of trypsin entrapped in electrospun poly ( $\epsilon$-caprolactone) nanofibers. Enzyme Microb. Technol. 2015, 79-80, 8-18.

37. Li, L.; Zhou, T.; Sun, G.; Li, Z.; Yang, W.; Jia, J.; Yang, G. Ultrasensitive electrospun nickel-doped carbon nanofibers electrode for sensing paracetamol and glucose. Electrochim. Acta 2015, 152, 31-37.

38. Su, Z.Q.; Li, J.F.; Li, Q.; Ni, T.Y.; Wei, G. Chain conformation, crystallization behavior, electrical and mechanical properties of electrospun polymer-carbon nanotube hybrid nanofibers with different orientations. Carbon 2012, 50, 5605-5617.

39. Jia, X.L.; Tang, T.H.; Cheng, D.; Guo, L.J.; Zhang, C.H.; Cai, Q.; Yang, X.P. Growth mechanism of bioglass nanoparticles in polyacrylonitrile-based carbon nanofibers. RSC Adv. 2014, 4, 64299-64309.

40. Shi, Z.; Chong, C.; Wang, J.; Wang, C.; Yu, X. Electrospun pitch/polyacrylonitrile composite carbon nanofibers as high performance anodes for lithium-ion batteries. Mater. Lett. 2015, 159, 341-344.

41. Song, K.L.; Wu, Q.L.; Zhang, Z.; Ren, S.X.; Lei, T.Z.; Negulescu, I.I.; Zhang, Q.G. Porous carbon nanofibers from electrospun biomass tar/polyacrylonitrile/silver hybrids as antimicrobial materials. ACS Appl. Mater. Interfaces 2015, 7, 15108-15116.

42. Wei, G.; Fan, X.; Liu, J.; Yan, C. Electrospun carbon nanofibers/electrocatalyst hybrids as asymmetric electrodes for vanadium redox flow battery. J. Power Sources 2015, 281, 1-6.

43. Nemati, M.; Voordouw, G. Modification of porous media permeability, using calcium carbonate produced enzymatically in situ. Enzyme Microb. Technol. 2003, 33, 635-642.

44. Kim, J.H.; Kim, C.H.; Yoon, H.; Youm, J.S.; Jung, Y.C.; Bunker, C.E.; Kim, Y.A.; Yang, K.S. Rationally engineered surface properties of carbon nanofibers for the enhanced supercapacitive performance of binary metal oxide nanosheets. J. Mater. Chem. A 2015, 3, 19867-19872.

45. Li, D.W.; Pang, Z.Y.; Chen, X.D.; Luo, L.; Cai, Y.B.; Wei, Q.F. A catechol biosensor based on electrospun carbon nanofibers. Beilstein J. Nanotechnol. 2014, 5, 346-354.

46. Li, H.H.; Zhao, S.; Gong, M.; Cui, C.H.; He, D.; Liang, H.W.; Wu, L.; Yu, S.H. Ultrathin PtPdTe Nanowires as Superior Catalysts for Methanol Electrooxidation. Angew. Chem. Int. Ed. 2013, 52, 7472-7476.

47. Kang, X.H.; Mai, Z.B.; Zou, X.Y.; Cai, P.X.; Mo, J.Y. Glucose biosensors based on platinum nanoparticles-deposited carbon nanotubes in sol-gel chitosan/silica hybrid. Talanta 2008, 74, 879-886.

48. Wang, G.; Pan, C.; Wang, L.P.; Dong, Q.; Yu, C.; Zhao, Z.B.; Qiu, J.S. Activated carbon nanofiber webs made by electrospinning for capacitive deionization. Electrochim. Acta 2012, 69, 65-70. 
49. Nia, P.M.; Lorestani, F.; Meng, W.P.; Alias, Y. A novel non-enzymatic $\mathrm{H}_{2} \mathrm{O}_{2}$ sensor based on polypyrrole nanofibers-silver nanoparticles decorated reduced graphene oxidenano composites. Appl. Surf. Sci. 2015, 332, 648-656.

50. Zhang, L.; Shi, Z.; Lang, Q.H.; Pan, J. Electrochemical synthesis of belt-like polyaniline network on $p$-phenylenediamine functionalized glassy carbon electrode and its use for the direct electrochemistry of horse heart cytochrome c. Electrochim. Acta 2010, 55, 641-647.

(C) 2015 by the authors; licensee MDPI, Basel, Switzerland. This article is an open access article distributed under the terms and conditions of the Creative Commons Attribution license (http://creativecommons.org/licenses/by/4.0/). 